\title{
Neutral and charged matter in equilibrium with black holes
}

\author{
K. A. Bronnikov \\ Center for Gravitation and Fundamental Metrology, \\ VNIIMS, 46 Ozyornaya Street, Moscow 119361, Russia, and \\ Institute of Gravitation and Cosmology, PFUR, \\ 6 Miklukho-Maklaya Street, Moscow 117198, Russia* \\ O. B. Zaslavskii \\ Department of Physics and Technology, \\ Kharkov V.N. Karazin National University, \\ 4 Svoboda Square, Kharkov, 61077, Ukraine ${ }^{\dagger}$
}

\begin{abstract}
We study the conditions of a possible static equilibrium between spherically symmetric, electrically charged or neutral black holes and ambient matter. The following kinds of matter are considered: (1) neutral and charged matter with a linear equation of state $p_{r}=w \rho$ (for neutral matter the results of our previous work are reproduced), (2) neutral and charged matter with $p_{r} \sim \rho^{m}, m>1$, and (3) the possible presence of a "vacuum fluid" (the cosmological constant or, more generally, anything that satisfies the equality $T_{0}^{0}=T_{1}^{1}$ at least at the horizon). We find a number of new cases of such an equilibrium, including those generalizing the well-known Majumdar-Papapetrou conditions for charged dust. It turns out, in particular, that ultraextremal black holes cannot be in equilibrium with any matter in the absence of a vacuum fluid; meanwhile, matter with $w>0$, if it is properly charged, can surround an extremal charged black hole.
\end{abstract}

PACS numbers: 04.70.Dy, 04.40.Nr, 04.70.Bw

\section{INTRODUCTION}

Black holes under real astrophysical conditions are always surrounded by some kind of matter which is either in equilibrium with the black hole or is falling on it. It is therefore of great interest to know the conditions of such an equilibrium and, in particular, which kinds of matter are compatible with it.

In our previous papers $[1,2]$ we studied the static equilibrium conditions between a black hole and ambient matter with the linear equation of state $p_{r}=w \rho$, where $\rho$ is the density, $w=$ const, and $p_{r}$ is the pressure in the direction orthogonal to the horizon (radial pressure in the case of spherical symmetry). It is well known that such an equilibrium is possible in the case $w=-1$, and examples of the corresponding space-times are those with a cosmological

*Electronic address: kb20@iyandex.ru

$\dagger$ Electronic address: zaslav@ukr.net 
constant and electromagnetic fields (e.g., the Reissner-Nordsrtröm-de Sitter space-time). According to $[1,2]$, an equilibrium is also possible for some other values of $w$, forming a discrete set $(w=-1 / 3$ in the generic case, which corresponds to disordered cosmic strings if we additionally suppose that matter is isotropic).

In [3], the same problem was considered for nonlinear equations of state of the type $p_{r}+\rho=w_{h}\left(\rho-\rho_{h}\right)^{\beta}$ where $\beta>1$ and $\rho_{h}$ is a nonzero density at the horizon. It was shown there that the results for isotropic and anisotropic fluids are very different. In the first case, the conditions for matter to be in equilibrium with the horizon are direct generalizations of those previously found [1]. In the second case, it turned out that there is no restriction on the equation of state but the horizon type is severely restricted: it should be simple only.

In this paper we reconsider this problem including matter with another type of nonlinear equations of state, $p_{r} \sim \rho^{m}, m>1$, which is of particular astrophysical interest since it includes polytropic equations of state. We also include into consideration electrically charged black holes and the possible electric charge of matter itself.

Although the electric charges of celestial bodies are usually negligible (see, e.g., [4]), this is probably true not for all objects; in particular, there are indications that neutron and quark stars can have quite significant charge to mass ratios and huge charges up to $10^{20}$ $\mathrm{C}$ [5], and there are attempts to build models of astrophysical objects where the electric charge plays a substantial role [6, 7]. Moreover, a significant electric charge is important for the formation of so-called quasiblack holes, systems which are on the threshold of forming a horizon (see [9] and references therein).

On the other hand, qualitative effects are possible which depend on the very existence of such a charge and taking place even at its very small values. In black hole physics, electric (or magnetic) charges can lead to results of general theoretical interest - such as the famous Majumdar-Papapetrou solutions [8] describing an equilibrium between charged extremal black holes and dustlike matter with the charge density $\rho_{e}= \pm \rho$ in proper units. Let us note that such an equilibrium (also important for quasiblack hole models) can be achieved even if a sphere of neutral hydrogen loses a fraction of $10^{-18}$ of its electrons.

It is also known that many features are qualitatively similar in rotating and electrically charged black holes. The first case is of greater interest in astrophysics but is more complicated. Therefore, it looks reasonable to study spherically symmetric configurations of Einstein-Maxwell systems to gain experience for future studies of rotating black holes.

The paper is organized as follows. In Sec. II we present the basic equations and conditions to be satisfied on a horizon. In Sec. III, we consider the following opportunities for matter near both charged and neutral spherically symmetric black holes: (1) neutral and charged matter with a linear equation of state $p_{r}=w \rho$ (for neutral matter the results of [1] are reproduced), (2) neutral and charged matter with $p_{r} \sim \rho^{m}, m>1$, and (3) the existence of a possible "vacuum fluid" (the cosmological constant or, more generally, anything that satisfies the condition $p_{r}^{\mathrm{vac}}+\rho^{\mathrm{vac}}=0$ [10], at least at the horizon) and its influence on the equilibrium conditions. We will find a number of new cases of such an equilibrium, in particular, those generalizing the Majumdar-Papapetrou conditions. Section IV is a conclusion. 


\section{BASIC EQUATIONS}

\section{A. Field equations}

Consider a generic spherically symmetric space-time with the metric (We use the units $c=\hbar=G=1)$

$$
d s^{2}=A(u) d t^{2}-\frac{d u^{2}}{A(u)}-r^{2}(u) d \Omega^{2} .
$$

Here, $u$ is the so-called quasiglobal coordinate especially suitable for dealing with Killing horizons because at such horizons it behaves as Kruskal-like coordinates in which the metric is manifestly regular there (see $[11,12]$ and references therein). For this reason, we can require analyticity of the metric at horizons in terms of $u$ (so that $A \sim u^{n}, n \in \mathbb{N}$ if $u=0$ is a horizon) remaining in the framework of a static metric.

We consider matter at rest, with the stress-energy tensor (SET)

$$
T_{\mu}^{\nu m}=\operatorname{diag}\left(\rho,-p_{r},-p_{\perp},-p_{\perp}\right),
$$

and a radial electric field with the SET

$$
T_{\mu}^{\nu \mathrm{em}}=\frac{e^{2}}{8 \pi r^{4}} \operatorname{diag}(1,1,-1,-1),
$$

where $e=e(u)$ is the charge inside a sphere of radius $r=r(u)$. The matter is supposed, in general, to be electrically charged, so the tensors (2) and (3) are not conserved each separately, but only their sum is.

The Maxwell equations read

$$
\frac{1}{\sqrt{-g}} \partial_{\mu}\left(F^{\mu \nu} \sqrt{-g}\right)=4 \pi j^{\mu}, \quad j^{\mu}=\rho_{e} u^{\mu},
$$

where $j^{\mu}$ is the current, $\rho_{e}$ is the invariant charge density, and $u^{\mu}$ the 4 -velocity of matter. For matter at rest,

$$
j^{\mu}=A^{-1 / 2} \rho_{e}(1,0,0,0) .
$$

Then, the only nontrivial component of the Maxwell equations has the form

$$
\left(F^{01} r^{2}\right)^{\prime}=4 \pi r^{2} \rho_{e} / \sqrt{A},
$$

where the prime stands for $d / d u$, and its solution is

$$
F^{01}=e(u) / r^{2},
$$

with

$$
e(u)=e_{\mathrm{bh}}+e_{m}(u), \quad e_{m}(u)=4 \pi \int_{0}^{u} \frac{d u}{\sqrt{A}} r^{2} \rho_{e},
$$

where we assume (without loss of generality) that $u=0$ is the event horizon of a black hole, $u>0$ outside it and $e_{\mathrm{bh}}$ is the black hole charge. The quantity $e_{m}(u)$ is the total charge of matter between the horizon and a sphere at given $u>0$. 
The conservation law can be written as follows:

$$
p_{r}^{\prime}+\frac{A^{\prime}}{2 A}\left(p_{r}+\rho\right)+\frac{2 r^{\prime}}{r}\left(p_{r}-p_{\perp}\right)-\frac{e \rho_{e}}{r^{2} \sqrt{A}}=0 .
$$

The Einstein equations give

$$
\begin{aligned}
G_{1}^{1}-G_{0}^{0} & =-2 A \frac{r^{\prime \prime}}{r}=8 \pi\left(p_{r}+\rho\right), \\
G_{1}^{1} & =\frac{1}{r^{2}}\left[-1+A^{\prime} r r^{\prime}+A r^{\prime 2}\right]=8 \pi p_{r}-\frac{e^{2}}{r^{4}}, \\
G_{0}^{0}-G_{2}^{2} & =\frac{1}{2 r^{2}}\left[-2+A\left(r^{2}\right)^{\prime \prime}-A^{\prime \prime} r^{2}\right]=-8 \pi\left(\rho+p_{\perp}\right)-\frac{2 e^{2}}{r^{4}}
\end{aligned}
$$

\section{B. Conditions near the horizon}

As already mentioned, we place a horizon at $u=0$; then, since it is by definition a regular surface, we require analyticity of the metric, and hence of the SET components, in terms of the quasiglobal coordinate $u$ at $u=0$. It then follows that, as $u \rightarrow 0$,

$$
\begin{aligned}
& A \approx A_{0} u^{n}, \quad n>0, \\
& \rho \approx \rho_{0} u^{k}, \quad k \geq 0,
\end{aligned}
$$

with integers $n$ and $k$. The pressure components must also behave as (14) with integer powers maybe other than $k$.

Eq. (10) leads to the well-known result that a nonzero density of matter at the horizon (that is, $k=0$ ) is only possible if $\rho+p_{r}=0$ or equivalently $T_{0}^{0}=T_{1}^{1}$, which characterizes the so-called "vacuum fluid" [10]. If this relation holds in the whole space or at least in a finite region, Eq. (10) takes the form $r^{\prime \prime}=0$, and (provided $r \neq$ const) after evident rescaling the quantity $r$ may be appointed the radial coordinate, $u=r$. The metric function $A(r)$ then remains arbitrary and can contain zeros (horizons) of any order; substitution of $A(r)$ into the Einstein equations and the conservation law yield expressions for $\rho(r)$ and $p_{\perp}(r)$. Moreover, if there is a radial electric field, it is natural to assume that the vacuum fluid itself is not charged, $\rho_{e}=0$, hence $e=e_{\mathrm{bh}}=$ const: the black hole can be charged, which does not prevent the procedure of obtaining a solution with given $A(r)$ because the electric field also satisfies the condition $T_{0}^{0}=T_{1}^{1}$.

Thus the situation with a vacuum fluid does not require a further study. It can happen, however, that $\rho+p_{r}=0$ only at the horizon, as is the case, e.g., in black hole solutions with scalar fields $[13]^{1}$ and horizons with such matter (to be called "pseudo-vacuum") deserve separate attention. Not being vacuum in general, such matter can be both neutral and charged.

Let us also adopt the following assumptions:

\footnotetext{
${ }^{1}$ For a minimally coupled scalar field $\phi$, the kinetic part of the SET $T_{\mu}^{\nu}$ is proportional to $A \phi^{\prime 2}$ and vanishes at horizons due to $A=0$, whereas the potential enters into the SET like the cosmological constant, i.e., is vacuum-like.
} 
(i) $\left|p_{r} / \rho\right|<C_{1}=$ const $<\infty,\left|p_{\perp} / \rho\right|<C_{2}=$ const $<\infty$, which is a weakened version of the dominant energy condition.

(ii) $\left|\rho_{e} / \rho\right|<C_{3}=$ const $<\infty$, and one can assume that near the horizon

$$
\rho_{e} \approx \alpha \rho u^{s} \sim u^{k+s}, \quad \alpha, s=\text { const, } \quad s \geq 0 .
$$

To sum up, we require regularity of the metric, so that it can be extended through the horizon, and, using the field equations, try to find out which kinds of matter are compatible with this regularity. We also assume that this matter is "not too exotic", that is, (i) the pressure to density ratio and (ii) the charge to mass density ratio are bounded above.

In what follows, we will distinguish (pseudo-)vacuum and ordinary matter. For ordinary matter, since $\rho+p_{r} \neq 0$ in general but $\rho+p_{r}=0$ at the horizon, we must require that both $\rho$ and $p_{r}$ vanish at the horizon, and in Eq. (14), $k>0$.

We will consider equilibrium conditions between a horizon and ambient matter in the following cases: (1) ordinary matter with $p_{r} \neq-\rho$ (neutral or charged, with linear and nonlinear equations of state), for which $k>0$ in (14), (2) pseudo-vacuum matter, for which $k=0$, and (3) a non-interacting mixture of ordinary and (pseudo-)vacuum matter.

\section{MATTER IN EQUILIBRIUM WITH THE HORIZON}

\section{A. Charged and neutral matter with a linear equation of state}

Suppose a linear equation of state for matter,

$$
p_{r}=w \rho, \quad w=\text { const } \neq-1 .
$$

After substitution to (9) we have

$$
2 A w \rho^{\prime}+A^{\prime}(w+1) \rho+2 \frac{r^{\prime}}{r}\left(p_{r}-p_{\perp}\right)-\frac{\sqrt{A} e \rho_{e}}{r^{2}}=0 .
$$

From (10) it now follows $\rho(0)=0$, so that $k>0$ and, even more than that, $k \geq n$ (generically $k=n$, while $k>n$ corresponds to $r^{\prime \prime}(0)=0$ ).

It is also clear from (15) and (8) that at small $u$

$$
e_{m} \sim u^{k-n / 2+s+1},
$$

and the exponent here is manifestly positive, so that, as should be the case in a regular configuration, $e_{m}(0)=0$.

Let us consider separately the cases of neutral $\left(e_{\mathrm{bh}}=0\right)$ and charged $\left(e_{\mathrm{bh}} \neq 0\right)$ black holes.

(a) $e_{\mathrm{bh}}=0$. It follows from (11) that $n=1$, since, in the order $O(1)$, the minus unity in the square brackets can be compensated only by the term $A^{\prime} r r^{\prime}$ and only in the case $n=1$, see [1]. 
In (17) the first two terms have the order $u^{n+k-1}$, the third one $\sim u^{n+k}$ and the fourth one

$$
e_{m} \rho_{e} \sqrt{A} \sim u^{2 k+2 s+1}
$$

Since $k \geq n$ and $s \geq 0$, the last two terms are negligible as compared to the first two, and we return to the old result for uncharged matter in the absence of a vacuum fluid [1]:

$$
w=-\frac{1}{1+2 k}
$$

In the generic case $k=n=1$ we obtain $w=-1 / 3$, which corresponds to a gas of disordered cosmic strings.

Thus near a neutral black hole a possible charge of matter itself has no effect.

(b) $e_{\mathrm{bh}} \neq 0$. Then, (9) gives

$$
[2 k w+n(w+1)] u^{k-1}+O\left(u^{k}\right)-\frac{2 \alpha e_{\mathrm{bh}}}{r_{h}^{2} \sqrt{A_{0}}} u^{k-n / 2+s}=0 .
$$

In the case of a simple horizon $(n=1)$, the last term in (21) is again negligible, and we return to the old condition (20). In addition, Eq. (11) in the order $O(1)$ leads to the relation

$$
-1+\left.r r^{\prime} A^{\prime}\right|_{u=0}+e_{\mathrm{bh}}^{2} / r_{h}^{2}=0
$$

Moreover, to provide a correct Taylor expansion in (21), we should require that $s$ should be half-integer.

In the case of a double horizon, $n=2$, Eqs. (11) and (12) lead to

$$
r_{h}=\left|e_{\mathrm{bh}}\right|, \quad A_{0}=1 / r_{h}^{2} .
$$

Substituting all this into (21) under the assumption $s=0$ (that is, $\rho_{e} / \rho=\alpha$ ), we obtain

$$
w=\frac{\alpha^{\prime}-1}{k+1}
$$

where $\alpha^{\prime}=\alpha \operatorname{sign} e_{\mathrm{bh}}$. In the generic case $k=n=2$, this gives $w=\left(\alpha^{\prime}-1\right) / 3$. In particular, if $\alpha^{\prime}=1$, then $w=0$, and we recover the well-known Majumdar-Papapetrou configuration of an extremal black hole and charged dust with $\left|\rho_{e}\right|=\rho$.

If matter and the black hole have charges of opposite signs, so that $\alpha^{\prime}<0$, then by $(24)$ we must have $w<-1 /(k+1)$.

There is one more opportunity with $n=2$ : if $s>0$, then the last term in (21) is negligible, and we obtain the same condition as for neutral matter in [1] for $n=2$, namely, $w=-1 /(1+k)$. In other words, a small charge density such that $\rho_{e} / \rho \rightarrow 0$ as $u \rightarrow 0$ is insignificant for the equilibrium conditions.

If we assume $n>2$, then Eq. (11) again gives $r_{h}^{2}=e_{\mathrm{bh}}^{2}$, but now in Eq. (12), in the order $O(1)$, we have $-1 / r_{h}^{2}$ on the left-hand side and $-2 / r_{h}^{2}$ on the right-hand side. Therefore the case $n>2$ is ruled out. 


\section{B. Neutral matter with a nonlinear equation of state}

Let us assume that $\rho_{e}=0$, hence $e_{m}=0$, but admit $e_{\mathrm{bh}} \neq 0$ (a charged black hole) and take the equation of state in the form

$$
p_{r}=B \rho^{m},
$$

where $m>1$, since $m<1$ would violate assumption (i) while $m=1$ has already been considered. We discard, in particular, all equations of state like that of Chaplygin gas ( $m<0$ since, as $\rho \rightarrow 0$, they give an infinite pressure incompatible with regularity of the horizon. It follows from (14) that $p_{r} \sim u^{m k}$, hence $m k$ should be a positive integer.

The form of the conservation law (9) now does not depend on whether the black hole is charged or not. Substituting in (9) $\rho \sim u^{k}$ and neglecting all terms of orders $o\left(u^{k-1}\right)$, we obtain

$$
B m \rho^{m-1} \rho^{\prime}+\frac{n}{2 u} \rho=0,
$$

and it follows from comparison of the exponents that

$$
(m-1) k+k-1=k-1 \Rightarrow(m-1) k=0,
$$

which is incompatible with our assumptions.

We see that neutral matter with the nonlinear equation of state (25), irrespective of the presence or absence of an electromagnetic field, cannot be in equilibrium with a black hole.

\section{Charged matter with a nonlinear equation of state}

Let us discuss the behavior of electrically charged matter with the equation of state (25). Neglecting the manifestly negligible terms, we can write the conservation law near the horizon as follows:

$$
B m \rho^{m-1} \rho^{\prime}+\frac{A^{\prime}}{2 A} \rho-\frac{e \rho_{e}}{r^{2} \sqrt{A}}=0 .
$$

We again consider separately the cases of charged and uncharged black holes.

(a) $e_{\mathrm{bh}}=0$. As in Sec. IIIA, it follows from (11) that $n=1$.

In (28), the orders of magnitude in the three terms are

$$
u^{k m-1}, \quad u^{k-1}, \quad u^{2 k+2 s},
$$

respectively, so that the second term, $O\left(u^{k-1}\right)$, is the largest and cannot be compensated by other terms. Thus charged nonlinear matter cannot be in equilibrium with a neutral black hole.

(b) $e_{\mathrm{bh}} \neq 0$. Now Eq. (11) does not restrict the order of the horizon. In (28), the first two terms are of the same orders of magnitude as in (29), but the third one is now $\sim u^{k+s-n / 2}$. Therefore an equilibrium is possible if

$$
n / 2-s=1
$$


A simple horizon $(n=1)$ is impossible since $s \geq 0$, a double horizon requires $s=0$ and so on.

For $n=2$, just as in Sec. III.A, Eqs. (11) and (12) lead to $e_{\mathrm{bh}}^{2}=r_{h}^{2}$ and $A_{0}=1 / r_{h}^{2}$. Then from (28) we find that

$$
\alpha=\operatorname{sign} e_{\mathrm{bh}}= \pm 1 \text {. }
$$

This means that a double horizon of a charged black hole can be in equilibrium with any matter obeying (25) $(m>1)$ whose charge density $\rho_{e}= \pm \rho$ has the same sign as the black hole. The only restriction on $m$ follows from the analyticity requirement for $p_{r}$ (see Sec. II.B) according to which $m k \in \mathbb{N}$.

Horizons of orders $n>2$ are impossible precisely for the same reason as in Sec. III.A.

\section{Pseudo-vacuum matter}

Suppose that near the horizon all relevant functions are represented by Taylor series whose first terms are

$$
\begin{aligned}
\rho & =\rho_{h}+c_{1} u^{a}+\ldots, \quad a=1,2, \ldots ; \\
p_{r} & =-\rho_{h}+w_{1}\left(\rho-\rho_{h}\right)^{q}+\ldots, \quad q=1,2, \ldots ; \\
p_{\perp} & =p_{r}+c_{2} u^{b}+\ldots, \quad b=0,1,2, \ldots,
\end{aligned}
$$

where $a$ and $q$ are generically equal to unity and $c_{1}, c_{2}, w_{1}$ are constants. A true vacuum fluid, in which $p_{r} \equiv-\rho$, corresponds to the special case $w_{1}=-1, q=1$. Moreover, a pseudo-vacuum matter can be charged, and then instead of Eq. (15) we have

$$
\rho_{e} \approx \alpha \rho_{h} u^{s} \Rightarrow e_{m} \sim u^{s+1}
$$

where $\alpha, s=$ const, $s \geq 0$.

The definitive property of vacuum matter is that the SET (34) preserves its form under arbitrary radial boosts [10], hence, unlike ordinary matter, it has no distinguished comoving reference frame. Examples of such matter are usual Maxwell radial electric and magnetic fields for which $p_{\perp}$ vac $=\rho^{\text {vac }}$, their analogs in nonlinear electrodynamics with Lagrangians of the form $L_{e}=L_{e}(F), F \equiv F_{\mu \nu} F^{\mu \nu}$ [14], Yang-Mills fields with a similar structure of the SET, and clouds of radially directed cosmic strings [15].

For matter that we here call pseudo-vacuum, the condition $\rho+p_{r}=0$ holds only at the horizon, and its properties are quite arbitrary in all the remaining space.

As already mentioned, a true vacuum fluid is compatible with horizons of any order. Pseudo-vacuum matter is much more general, as is seen, in particular, from (32) and (33), therefore it is clear that any horizons can be in equilibrium with it, and a detailed analysis along the same lines as before reveals more than a dozen of particular forms of such an equilibrium. We, however, mention here just two features that can be of certain interest:

- Anisotropy of pseudo-vacuum matter is required to provide equilibrium with horizons of orders $n>2$. 
- In all cases where the matter charge density is significant for maintaining the equilibrium, we have $s>0$, i.e., the density $\rho_{e}$ vanishes at the horizon (though slowly enough).

\section{E. A non-interacting mixture of ordinary and (pseudo) vacuum matter}

Let us now find out what changes in our conclusions made in Sections 3.1-3.3 if we add to the system described there a vacuum or pseudo-vacuum fluid with the SET

$$
T_{\mu}^{\nu \mathrm{vac}}=\operatorname{diag}\left(\rho^{\mathrm{vac}}, \rho^{\mathrm{vac}},-p_{\perp}^{\mathrm{vac}},-p_{\perp}{ }^{\mathrm{vac}}\right),
$$

and there is no interaction between the SETs $T_{\mu}^{\nu m}+T_{\mu}^{\nu \mathrm{em}}$ and $T_{\mu}^{\nu \mathrm{vac}}$, that is, the conservation law (9) holds, and we also have $\nabla_{\nu} T_{\mu}^{\nu \mathrm{vac}}=0 .^{2}$ If the SET (34) is added to (2) and (3), the conservation law (9) for matter under consideration and the Einstein equation (10) do not change (and (10) again leads to $k \geq n$ ), while (11) and (12) take the form

$$
\begin{gathered}
G_{1}^{1}=\frac{1}{r^{2}}\left[-1+A^{\prime} r r^{\prime}+A r^{2}\right]=8 \pi p_{r}-\frac{e^{2}}{r^{4}}-8 \pi \rho^{\mathrm{vac}}, \\
G_{0}^{0}-G_{2}^{2}=\frac{1}{2 r^{2}}\left[-2+A\left(r^{2}\right)^{\prime \prime}-A^{\prime \prime} r^{2}\right]=-8 \pi\left(\rho+p_{\perp}\right)-\frac{2 e^{2}}{r^{4}}-8 \pi\left(\rho^{\mathrm{vac}}+p_{\perp}{ }^{\mathrm{vac}}\right) .
\end{gathered}
$$

Eq. (35) in the order $O(1)$ gives

$$
1-\left.A^{\prime} r r^{\prime}\right|_{u=0}=e_{\mathrm{bh}}^{2} / r_{h}^{2}+\Lambda r_{h}^{2}, \quad \Lambda:=\left.8 \pi \rho^{\mathrm{vac}}\right|_{u=0} .
$$

Therefore higher-order horizons $(n>1)$, such that $A^{\prime}(0)=0$, become possible even for $e_{\mathrm{bh}}=0$.

Eq. (36) in the same order of magnitude leads to

$$
2+\left.A^{\prime \prime} r^{2}\right|_{u=0}=4 e_{\mathrm{bh}}^{2} / r_{h}^{2}+2 \Lambda_{*} r_{h}^{2}, \quad \Lambda_{*} ;=\left.8 \pi\left(\rho^{\mathrm{vac}}+p_{\perp}^{\mathrm{vac}}\right)\right|_{u=0} .
$$

Now it is easy to obtain equilibrium conditions in the presence of vacuum matter.

(a) The equation of state (16), $e_{\mathrm{bh}}=0$ (a neutral black hole). Horizons of any order $n$ are possible, and (9) gives

$$
w=-\frac{n}{2 k+n},
$$

reproducing the result of [1]. Eqs. (37) and (38) lead to relations between the parameters of the configuration. For $n>1$ we obtain

$$
\Lambda r_{h}^{2}=1, \quad\left(2 \Lambda_{*}-A^{\prime \prime}(0)\right) r_{h}^{2}=2,
$$

\footnotetext{
${ }^{2}$ In our reasoning here, a difference between vacuum and pseudo-vacuum matter is insignificant because only the values of its density and pressures at the horizon will be relevant.
} 
and the second relation indicates that horizons of order $n>2$ are only possible if $\Lambda_{*}>0$. The cosmological constant, for which $p_{\perp}{ }^{\mathrm{vac}}=p_{r}{ }^{\mathrm{vac}}=-\rho^{\mathrm{vac}}$, is thus excluded.

A possible nonzero $\rho_{e}$ is insignificant.

(b) The equation of state (16), $e_{\mathrm{bh}} \neq 0$. Again horizons of any order are possible; Eq. (21) is again valid and leads to

$$
s \geq n / 2-1 \text {. }
$$

In the case of a simple horizon, as before, the last term in (9) is insignificant, so that for $w$ we have the old result (20), and it is required that $s$ should be half-integer.

For $n \geq 2$ and the exact equality in (41) (in particular, $s=0$ for $n=2$ ), we obtain instead of (24) the relation

$$
w=\frac{2 \alpha_{*}-n}{2 k+n}, \quad \alpha_{*}:=\frac{\alpha e_{\mathrm{bh}}}{r_{h}^{2} \sqrt{A_{0}}}
$$

while due to (37) and (38) the parameters of the configuration are related by

$$
e_{\mathrm{bh}}^{2} / r_{h}^{2}=1-\Lambda r_{h}^{2}, \quad \delta_{n 2} A_{0} r_{h}^{2}=1+r_{h}^{2}\left(\Lambda_{*}-2 \Lambda\right)
$$

(the Kronecker symbol $\delta_{n 2}$ indicates that this term appears only if $n=2$ ).

If (41) is a strict inequality (i.e., our matter is weakly charged), we must put $\alpha_{*}=0$ in (42) and thus return to the old expression (39).

(c) Neutral matter with the equation of state (25). The conclusion made in Sec. III.B, that such matter cannot be in equilibrium with any black hole, rests solely on Eq. (9) and therefore does not change in the presence of a vacuum fluid.

(d) Charged matter with the equation of state (25). As in Sec. III.C, the conservation law near the horizon leads to (28) which again discards simple horizons and yields $s=n / 2-1$ for higher-order horizons $(n \geq 2)$.

Unlike Sec. III.C, horizons of any order $n \geq 2$ now become possible, the system parameters obeying the relations (43), and instead of (31) we now find the condition

$$
\alpha_{*}=n / 2 \text {. }
$$

The power $m$ in (25) remains arbitrary but must obey the condition $m k \in \mathbb{N}$ to maintain analyticity.

\section{CONCLUSION}

Our main results are summarized in the following table. 
Table: Matter equilibrium conditions for neutral and charged black holes with horizons of order $n$. The symbol "vac" denotes (pseudo)vacuum matter, the symbol $\oplus$ a non-interacting mixture, and $\{s\}$ is the fractional part of $s$. All other notations can be found in the text.

\begin{tabular}{|c|c|c|}
\hline Matter & $e_{\mathrm{bh}}=0$ & $e_{\mathrm{bh}} \neq 0$ \\
\hline vac & $\forall n$ & $\forall n$ \\
\hline$p_{r}=w \rho$ & $n=1, \quad w=-\frac{1}{1+2 k}$ & $\begin{array}{l}n=1, \quad w=-\frac{1}{1+2 k}, \quad\{s\}=1 / 2 \\
n=2, \quad w=\frac{\alpha^{\prime}-1}{1+2 k}, \quad s=0 \\
n=2, \quad w=-\frac{1}{1+k}, \quad\{s\}=1 / 2\end{array}$ \\
\hline $\operatorname{vac} \oplus\left(p_{r}=w \rho\right)$ & $\forall n, \quad w=-\frac{n}{2 k+n}$ & $\begin{array}{l}n=1, \quad w=-\frac{1}{1+2 k}, \quad\{s\}=1 / 2 \\
n \geq 2, \quad w=-\frac{2 \alpha_{*}-n}{1+2 k}, \quad s=n / 2-1\end{array}$ \\
\hline$p_{r}=B \rho^{m}, m>1$ & none & $n=2, \quad \alpha= \pm 1, \quad s=0$ \\
\hline vac $\oplus\left(p_{r}=B \rho^{m}, m>1\right)$ & none & $n \geq 2, \quad \alpha_{*}=n / 2, \quad s=n / 2-1$ \\
\hline
\end{tabular}

Thus, in the framework of static, spherically symmetric configurations in general relativity, we have analyzed the equilibrium conditions between an electrically charged or neutral black hole and ambient matter. To do so, we used near-horizon expansions of the Einstein equations and the conservation law for matter. Actually, all the conditions were obtained from the largest terms in such expansions; in general, smaller terms do not lead to new restrictions but only provide relations between further expansion factors of the corresponding functions of the radial coordinate.

For linear equations of state of the form $p_{r}=w \rho$, we have confirmed our previous results [1] that in the case of neutral matter an equilibrium is possible for a discrete set of values of $w$ between zero and $-1 / 3(w=-1 / 3$ is the generic case, corresponding to a distribution of disordered cosmic strings if the matter is isotropic). We have found out how these conditions are modified in the presence of an electric charge. It was stressed in [1] that inside a "normal" star (i.e., with everywhere nonnegative pressure) a black hole cannot exist. However, as follows from the present results, overcharged normal matter $(w>0, \alpha>1)$ can be in equilibrium with a black hole but its horizon must be extremal $(n=2)$.

Neutral matter with a nonlinear equation of state (25) cannot be in equilibrium with any black hole. However, charged matter with the same equation of state can surround extremal charged black holes under the same condition on the charge density as is known for the Majumdar-Papapetrou solutions.

Some additional possibilities of this kind are opened if vacuum (or pseudo-vacuum) matter is added to the system, and in particular, only in its presence horizons of orders $n>2$ (ultraextremal) become possible.

Thus inclusion of an electric charge (however small it be) into consideration leads to qualitatively new possibilities for both linear and nonlinear equations of state which were absent in the neutral case. 
Our reasoning has been entirely local and relied on near-horizon expansions, thus no assumptions on global or asymptotic properties of space-time have been made. We also did not assume any particular equation of state for matter and even did not restrict the behavior of the transverse pressure except for its regularity requirement. In this sense, our conclusions are model-independent.

\section{Acknowledgment}

The authors thank José P.S. Lemos for a helpful discussion. The work of K.B. was supported in part by the Russian Foundation for Basic Research Grant No. 09-02-00677a, by NPK MU grant at PFUR, and by FTsP "Nauchnye i nauchno-pedagogicheskie kadry innovatsionnoy Rossii" for the years 2009-2013.

[1] K.A. Bronnikov and O.B. Zaslavskii, Phys. Rev. D 78, 021501(R) (2008).

[2] K.A. Bronnikov and O.B. Zaslavskii, Class. Quantum Grav. 26, 165004 (2009).

[3] O.B. Zaslavskii, Phys. Rev. D 81, 107501 (2010).

[4] A. Baushev and P. Chardonnet, Electric charge estimation of a new-born black hole, Int. J. Mod. Phys. D 18, 2035 (2009); ArXiv: 0905.4071.

[5] M. Malheiro, R. Picanco, S. Ray, J.P.S. Lemos, and V.T. Zanchin, Int. J. Mod. Phys. D 13, 1375 (2004).

[6] S. Thirukkanesh and S.D. Maharaj, Class. Quantum Grav. 25, 235001 (2008).

[7] V. Varela, F. Rahaman, S. Ray, K. Chakraborty, and M. Kalam, Phys. Rev. D 82, 044052 (2010).

[8] S.D. Majumdar, Phys. Rev. 72, 390 (1947);

A. Papapetrou, Proc. R. Irish Acad., Sect. A 51, 191 (1947).

[9] José P.S. Lemos and O.B. Zaslavskii, Phys. Rev. D 76, 084030 (2007).

[10] I.G. Dymnikova, Gen. Rel. Grav. 24, 235 (1992); Class. Quantum Grav. 19, 225 (2002); Int. J. Mod. Phys. D 12, 1015 (2003).

[11] K.A. Bronnikov and S.G. Rubin, Lectures on Gravitation and Cosmology (MIFI publishers, Moscow, 2008, in Russian).

[12] K.A. Bronnikov, E. Elizalde, S.D. Odintsov, and O.B. Zaslavskii, Phys. Rev. D 78, 064049 (2008); Arxiv: 0805.1095.

[13] K.A. Bronnikov and G.N. Shikin, Grav. Cosmol. 8, 107 (2002), gr-qc/0109027;

K.C.K. Chan, J.H. Horne, and R.B. Mann, Nucl. Phys. B 447, 441 (1995);

U. Nucamendi and M. Salgado, Phys. Rev. D 68, 044026 (2003);

O. Bechmann and O. Lechtenfeld, Class. Quantum Grav. 12, 1473 (1995);

H. Dennhardt and O. Lechtenfeld, Int. J. Mod. Phys. A 13, 741 (1998);

K.A. Bronnikov and J.C. Fabris, Phys. Rev. Lett. 96, 251101 (2006).

[14] K.A. Bronnikov, Phys. Rev. D 63, 044005 (2001).

[15] P.S. Letelier, Phys. Rev. D 20, 1294 (1979). 